\title{
Monte Carlo Simulations of a Scanning System Based on a Panoramic X-Ray Tube with a Conical Anode
}

\author{
Andrii Sofiienko, ${ }^{1}$ Chad Jarvis, ${ }^{2}$ and Ådne Voll ${ }^{3}$ \\ ${ }^{1}$ University of Bergen, Allegaten 55, P.O. Box 7803, 5020 Bergen, Norway \\ ${ }^{2}$ Christian Michelsen Research AS, Fantoftveien 38, P.O. Box 6031, 5892 Bergen, Norway \\ ${ }^{3}$ Visuray AS, Strandbakken 10, 4070 Randaberg, Norway \\ Correspondence should be addressed to Andrii Sofiienko; asofienko@gmail.com
}

Received 9 May 2014; Accepted 30 July 2014; Published 21 August 2014

Academic Editor: Zhiqiang Mao

Copyright (C) 2014 Andrii Sofiienko et al. This is an open access article distributed under the Creative Commons Attribution License, which permits unrestricted use, distribution, and reproduction in any medium, provided the original work is properly cited.

\begin{abstract}
Monte Carlo simulations were used to study photon production in a panoramic X-ray tube with a conical tungsten target to determine the optimal characteristics of the target shape and electron beam configuration. Several simulations were performed for accelerating potentials equal to $250 \mathrm{kV}, 300 \mathrm{kV}$, and $500 \mathrm{kV}$ with electron beams of various radii and anode sizes. The angular distribution of the photon intensity was analysed by numerical calculations for an assembly composed of an X-ray tube and an external collimator with a cylindrical hole to simulate a panoramic scanning system with an X-ray pencil beam.
\end{abstract}

\section{Introduction}

$\mathrm{X}$-ray techniques are commonly used in the nondestructive testing [1] of industrial parts, material characterisation, security, many medical applications [2-4], and the examination of various other types of specimens $[5,6]$. The most common techniques used for obtaining images are radioscopy for $2 \mathrm{D}$ imaging and computed tomography for 3D imaging. Additionally, scanning systems based on a pencil-beam technique [1], where an X-ray tube uses either an inclined massive target or a conical target for panoramic scanning, are also commonly used. X-ray tubes with a conical target $[7,8]$ have the simplest design for $360^{\circ}$ panoramic scanning systems [9] and can be used, for example, to inspect oil wells and pipes. For scanning technology, maximising the output X-ray intensity in the collimated beam is important. Variations in the electron beam diameter, the shape of the conical target, and the inclination angle of the output collimated X-ray beam can significantly affect the output X-ray intensity in the collimated beam and limit the performance of the scanning system. However, the literature shows a lack of detailed information published about the effect of the geometrical parameters of an electron beam and a conical target on the intensity of the angular distribution of collimated X-rays in the $250-500 \mathrm{kV}$ range of accelerating potentials (which is interesting for industrial applications in which the panoramic scanning technique is used). The general purpose of this study was to investigate, using Monte Carlo simulations, the $\mathrm{X}$-ray generation process in an X-ray tube with a conical tungsten anode and cylindrical symmetry. We determined the following parameters for use in practical applications: the intensity of generated X-rays in the energy range of 250$500 \mathrm{keV}$, the optimal target shape, the angular distribution of the intensity of the generated X-rays, the intensity of collimated photons through a cylindrical hole from an external collimator, and the efficiency of X-ray generation.

\section{Description of the Monte Carlo Simulations}

Monte Carlo (MC) simulations were performed using the software suite Xenos by Field Precision [10]. The specific program used to generate the Monte Carlo simulations is called GamBet that uses conformal meshes for 2D/3D Monte Carlo simulations of radiation transport in matter [10]. The unitized code suite handles the full range of electron, photon, and positron interactions with high accuracy. Applications include X-ray targets, electron-beam heating, radiographic 


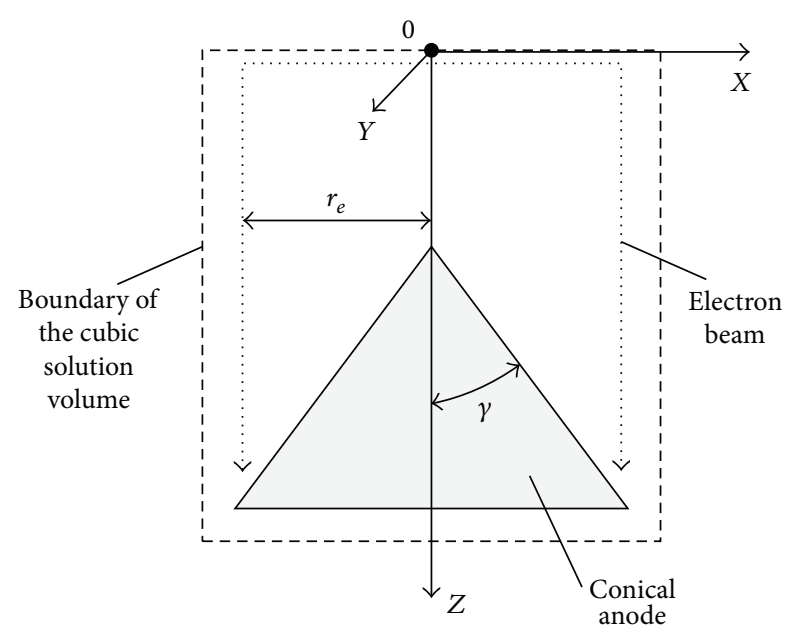

FIgURE 1: A geometrical model of the conical tungsten target and electron beam that were used for the MC simulations.

imaging, positron physics, radiation therapy research, and shielding design. GamBet combines Field Precision's finiteelement codes with the package PENELOPE [11] and claims the maximal simulation error of $15 \%$. PENELOPE is a public program used to perform MC simulations of electron-photon transport in various materials. The solution volume for the object mesh had different ranges for the different sizes of the tungsten conical anode (the volume depends on the apex angle of the conical anode $2 \gamma$, Figure 1 ) and the different radii of the electron beam $\left(r_{e}\right)$ considered. Table 1 provides information about the solution volume for the mesh for each simulation and the main geometrical parameters. The tungsten conical anode was centred at $(0.0 \mathrm{~mm}, 0.0 \mathrm{~mm})$ in the $X Y$ plane. MC simulations were performed for the geometries presented in Table 1 and Figure 1, at electron energies of $250 \mathrm{keV}, 300 \mathrm{keV}$, and $500 \mathrm{keV}$. The number of high-energy electrons was the same for all simulations: $N_{e}=$ $4.613 \cdot 10^{5}$. The height of the cone was different for different radii of the electron beam and apex angles of the cone because the radius of the base of the cone was the same as the radius of the electron beam for all simulations, as shown in Figure 1.

The radial distribution of electrons in an electron beam was modelled as a Gaussian function of the diameter of the electron beam $\left(D_{e}\right)$ :

$$
f_{e}\left(x, y, D_{e}\right)=\frac{1}{2 \pi \cdot\left(D_{e} / 4\right)^{2}} \cdot \exp \left(-\frac{x^{2}+y^{2}}{2 \cdot\left(D_{e} / 4\right)^{2}}\right) .
$$

This electron distribution corresponds to a $90 \%$ probability of any electrons occurring within an electron beam with a diameter $D_{e}$.

\section{Results and Discussion}

3.1. The Energy Distribution of Generated Photons. X-ray spectra were generated using an MC generator with electron energies equal to $250 \mathrm{keV}, 300 \mathrm{keV}$, and $500 \mathrm{keV}$. These spectra were created to investigate the effect of the shape of

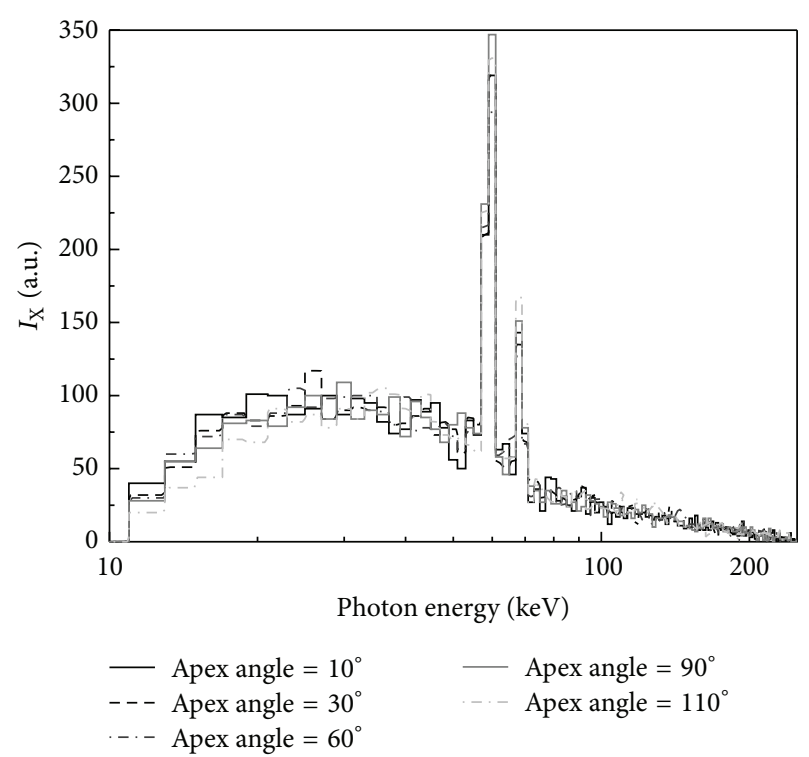

Figure 2: The energy spectra of X-ray photons generated at an electron energy of $250 \mathrm{keV}$ and at different values of the apex angle of the conical anode; $r_{e}=2 \mathrm{~mm}$.

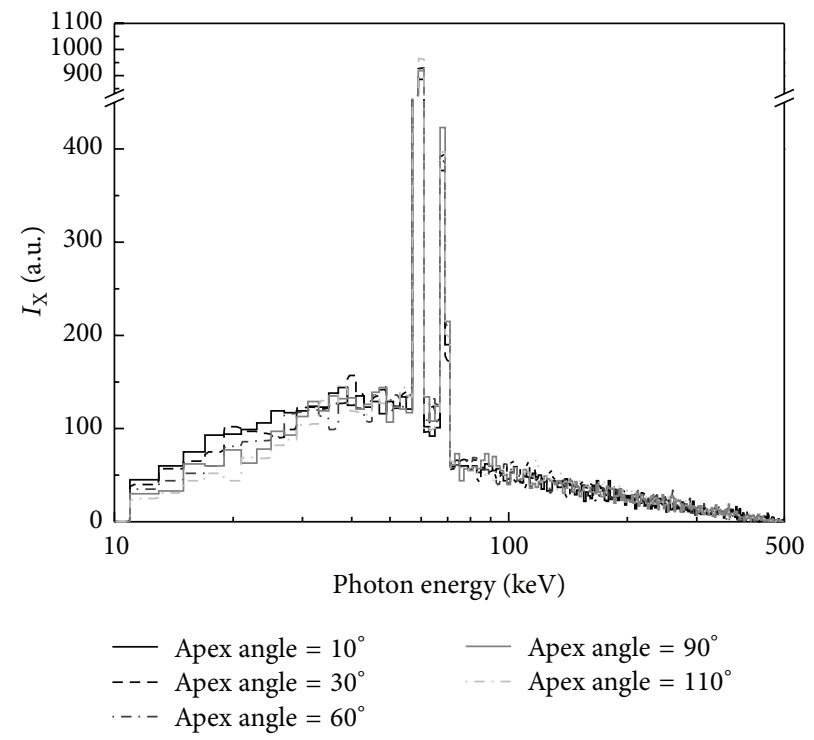

FIgURE 3: The energy spectra of X-ray photons generated at the electron energy of $500 \mathrm{keV}$ and at different values of the apex angle of the conical anode; $r_{e}=2 \mathrm{~mm}$.

the conical anode (target) and the electron beam size on the efficiency of X-ray generation and on the angular distribution of the flux of generated photons. The energy distributions of all of the generated X-rays (which were emitted in different directions after being generated but with the same energy within an energy bin of $2 \mathrm{keV}$ ) obtained when the electron energy was equal to $250 \mathrm{keV}$ and $500 \mathrm{keV}$ are shown in Figures 2 and 3 for conical anodes with different apex angles and for the $4 \mathrm{~mm}$ diameter electron beam. The energy threshold was of $14 \mathrm{keV}$. 
TABLE 1: Main geometrical parameters of the MC simulations.

\begin{tabular}{|c|c|c|c|c|c|}
\hline$r_{e}, \mathrm{~mm}$ & $2 \gamma$, degrees & $x$-axis, $\mathrm{mm}$ & $y$-axis, $\mathrm{mm}$ & $z$-axis, $\mathrm{mm}$ & Spatial resolution, $\mu \mathrm{m}$ \\
\hline \multirow{5}{*}{0.5} & 10 & \multirow{5}{*}[-0.6;0.6]{} & \multirow{5}{*}[-0.6;0.6]{} & {$[0 ; 6]$} & \multirow{5}{*}{5.0} \\
\hline & 30 & & & {$[0 ; 3]$} & \\
\hline & 60 & & & {$[0 ; 1.5]$} & \\
\hline & 90 & & & {$[0 ; 1.0]$} & \\
\hline & 110 & & & {$[0 ; 1.0]$} & \\
\hline \multirow{5}{*}{2.0} & 10 & \multirow{5}{*}[-2.2;2.2]{} & \multirow{5}{*}[-2.2;2.2]{} & {$[0 ; 23]$} & \multirow{5}{*}{15} \\
\hline & 30 & & & {$[0 ; 10]$} & \\
\hline & 60 & & & {$[0 ; 5]$} & \\
\hline & 90 & & & {$[0 ; 3]$} & \\
\hline & 110 & & & {$[0 ; 3]$} & \\
\hline \multirow{5}{*}{5.0} & 10 & \multirow{5}{*}[-5.1;5.1]{} & \multirow{5}{*}[-5.1;5.1]{} & {$[0 ; 60]$} & \multirow{5}{*}{35} \\
\hline & 30 & & & {$[0 ; 20]$} & \\
\hline & 60 & & & {$[0 ; 10]$} & \\
\hline & 90 & & & {$[0 ; 10]$} & \\
\hline & 110 & & & {$[0 ; 5]$} & \\
\hline
\end{tabular}

It was observed that the shape of the X-ray spectrum of all of the generated photons is the same for different electron beam radii. As can be seen from Figures 2 and 3, there are some differences in the intensity of generated $\mathrm{X}$-ray spectra in the range of energies up to approximately $50 \mathrm{keV}$ for the different types of conical anodes, which showed different apex angle values. These differences occurred because the generated photons had a larger attenuation depth for larger values of the apex angle $2 \gamma$. This effect may also depend on the angular distribution of the generated photons, but it has a small influence on the total intensity of the generated photons within $1.0-1.2 \%$ for all simulations at different electron energies.

3.2. Angular Distribution of the Flux of Generated X-Rays. The spatial positions of the generated photons $\left(x_{i}, y_{i}, z_{i}\right)$ after the MC simulation were distributed on an external rectangular surface (the conical anode was located inside this cubic solution volume), which was determined by programming a mesh for the volume of simulated objects. To investigate the angular distribution of the photons in cylindrical geometry (which is more useful for the panoramic scanning technique), it is necessary to translate the initial spatial positions of the photons on the surface of the external cylinder into a radius $R_{c}$ (the value of $10 \mathrm{~mm}$ was used). This translation can be performed in the following way. First, we form a ray directed from the initial position of a photon on the rectangular surface to a second position on the cylindrical surface using the following linear coordinate transformation:

$$
\begin{aligned}
& x_{2 i}=x_{i}+x_{d i} t_{i} \\
& y_{2 i}=y_{i}+y_{d i} t_{i} \\
& z_{2 i}=z_{i}+z_{d i} t_{i},
\end{aligned}
$$

where the direction vector $\left(x_{d i}, y_{d i}, z_{d i}\right)$ is formed by the normalised components of the momentum vector $\left(p_{x i}, p_{y i}, p_{z i}\right)$.
Then, we substitute the ray into the parametric equation for a cylinder:

$$
x_{2 i}^{2}+y_{2 i}^{2}=R_{c}^{2}
$$

Next, we solve for $t$ :

$t_{i}$

$$
=\frac{x_{i} x_{d i}+y_{i} y_{d i}}{x_{d i}^{2}+y_{d i}^{2}} \cdot\left(\sqrt{1-\frac{\left(x_{d i}^{2}+y_{d i}^{2}\right)\left(x_{i}^{2}+y_{i}^{2}-R_{c}^{2}\right)}{\left(x_{i} x_{d i}+y_{i} y_{d i}\right)^{2}}}-1\right) .
$$

Lastly, we substitute the value of $t$ back into the equations in formula (1), which give the new coordinates $\left(x_{2 i}, y_{2 i}, z_{2 i}\right)$ of the photons on the surface of the external cylinder. A schematic showing the measurement of the photons on the external cylindrical surface is shown in Figure 4. For each direction from the top of the conical anode with an inclination angle $\alpha_{j}$ relative to the vertical symmetry axis, the area of the cylindrical sector on the cylindrical surface $\left(\Delta S_{j}\right)$ was calculated for a constant value of the output angle $\Delta \theta=4^{\circ}$, and the number of photons $\left(\Delta N_{j}\right)$ was calculated within this area of the cylindrical sector, making it possible to calculate the angular distribution of the flux of the generated photons as follows:

$$
\begin{gathered}
\Phi\left(\alpha_{j}\right)=\frac{\Delta N_{j}\left(\alpha_{j}, \Delta \theta\right)}{\Delta S_{j}\left(\alpha_{j}, \Delta \theta\right) \Delta t_{s}}, \\
\Delta S_{j}\left(\alpha_{j}, \Delta \theta\right) \\
=2 \pi \cdot R_{c}^{2} \cdot\left(\cot \left(\alpha_{j}-\frac{\Delta \theta}{2}\right)-\cot \left(\alpha_{j}+\frac{\Delta \theta}{2}\right)\right),
\end{gathered}
$$

where $\Delta t_{s}$ is a normalisation time that depends on the number of the electrons in the beam $\left(N_{e}\right)$ and on the electrical 


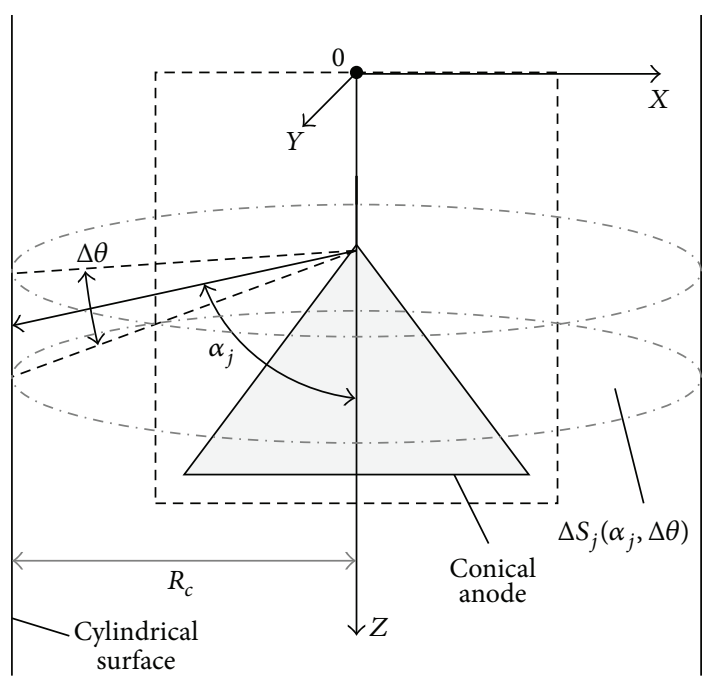

FIGURE 4: A schematic showing the measurement of the angular distribution of the photon flux in the cylindrical coordinate system.

power $\left(W_{T}\right)$ of the electron beam: $\Delta t_{s}=e N_{e} U_{T} / W_{T}$, where $e$ is the elementary electron charge and $U_{T}$ is the accelerating potential of the X-ray tube.

The angular distributions of the flux of generated photons calculated from the MC simulations are shown in Figure 5 for an electron energy of $250 \mathrm{keV}$ and $r_{e}=2 \mathrm{~mm}$. The step size of the inclination angle $\alpha_{j}$ was $\Delta \alpha=7^{\circ}$. For all cases, the electrical power of the electron beam was normalised to 1.0 Watts to obtain useful values of the photon flux for comparison with real-world values.

As shown in Figure 5, the angular distribution of the generated photons is different for different radii of the electron beam and shapes of the conical anode. It was also observed that the shape is different for different electron energies. The maximum value of the photon flux at an inclination angle $\alpha_{j m}$ is one of the most important parameters in this case. The relationships between these parameters are shown in Figures 6,7 , and 8 for different electron energies and geometries.

The errors in the inclination angles $\alpha_{j m}$ were estimated with the step size $\Delta \alpha=7^{\circ}$ that was used to calculate the area of the cylindrical sector on the external cylindrical surface $\left(\Delta S_{j}\right.$, formula (6)). The errors in the maximum values of the photon flux $\left(\Phi_{x m}\right.$, at an inclination angle $\left.\alpha_{j m}\right)$ were calculated as probable statistical deviations in the number of photons $\left(\Delta N_{j}\right)[12]$ that were passed through the cylindrical sector $\Delta S_{j}$ using the following relation:

$$
\Delta \Phi_{x m}\left(\alpha_{j m}\right)=\frac{\Phi_{x m}\left(\alpha_{j m}\right)}{\sqrt{\Delta N_{j}\left(\alpha_{j m}, \Delta \theta\right)}} .
$$

The results, presented in Figures 6-8, allow for the selection of the optimal value of the anode apex angle or the inclination angle for the output X-ray flux if one of these parameters is known. As indicated by the simulations, an accelerating potential in the range of $250-500 \mathrm{kV}$ does not significantly affect the maximum flux of generated $\mathrm{X}$-rays at the same

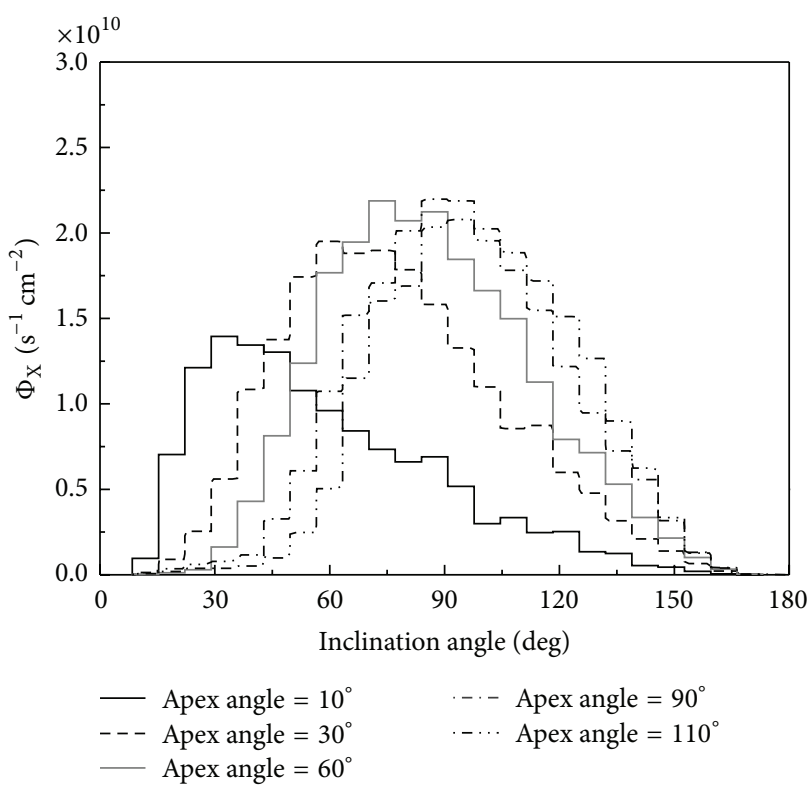

Figure 5: The angular distributions of the flux of generated X-ray photons in cylindrical geometry at different values of the apex angle of the anode for an electron energy equal to $250 \mathrm{keV}$ and an electron beam radius equal to $2.0 \mathrm{~mm}$.

electrical power and at the same radial distance from the symmetry axis of the conical anode. The probable variations are located within the range of the estimated numerical errors. The increase in the flux of generated photons at higher accelerating potentials of the X-ray tube may be due to the dependence of the efficiency of photon generation on the electron energy. This efficiency can be estimated for bremsstrahlung radiation using the following empirical relation [13]:

$$
\eta_{X} \approx(8 \pm 2) \cdot 10^{-10} Z E_{e},
$$

where the electron energy $E_{e}$ has units of "eV." As indicated by formula (8), the increase in the electron energy (or accelerating potential) linearly affects the efficiency of photon generation. However, the range of high-energy electrons in the target medium is also dependent on their energy [14]. Thus, the X-ray photons, generated by electrons with a greater energy, will have a greater attenuation distance inside the target, which will have an exponential effect on the output photon flux.

3.3. The Angular Distribution of Photon Intensity in Collimated $X$-Ray Beam. To simplify the MC simulations, which require a large amount of computation time, the following simple numerical method was used to simulate an external cylindrical collimator with any thickness $\Delta R$. For this task, it is necessary to know the distribution of the generated photons on the first surface of the collimator (with radius $R_{c}$ ) and that on the second surface (with radius $R_{c}+\Delta R$ ). These distributions can be calculated using a second translation of the spatial positions of the photons on the surface with radius $R_{c}$ to the surface with new radius $R_{c}+\Delta R$; moreover, 


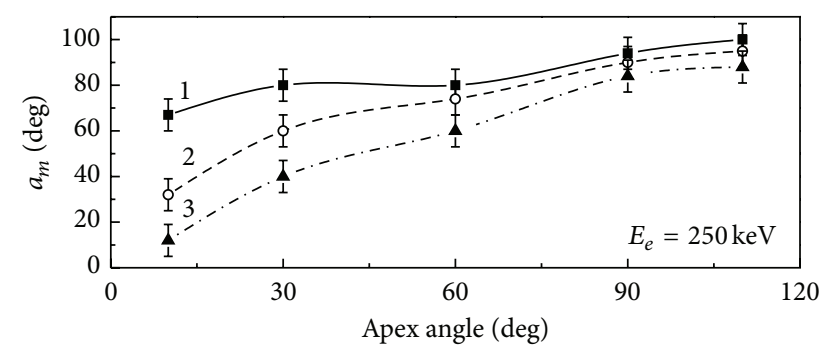

(a)

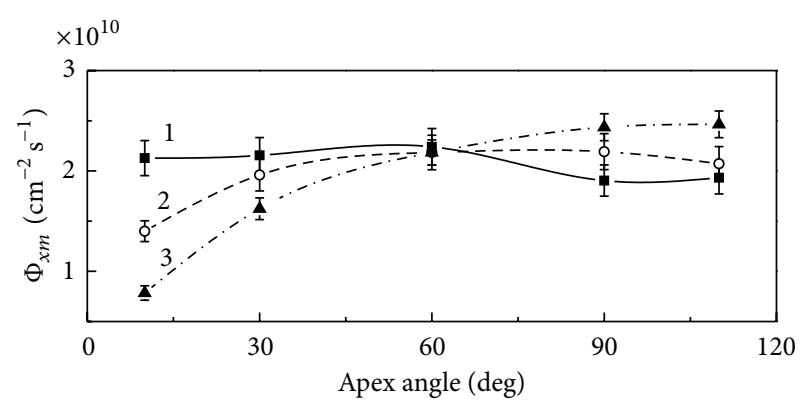

(b)

Figure 6: The dependences of the inclination angle, associated with the maximum value of the flux of generated X-rays (a), and the maximum value of the flux of generated X-rays (b) on the value of the apex angle of the conical anode at different electron beam radii: $0.5 \mathrm{~mm}(1), 2.0 \mathrm{~mm}$ (2), and $5.0 \mathrm{~mm}$ (3). The electron energy is $250 \mathrm{keV}$.

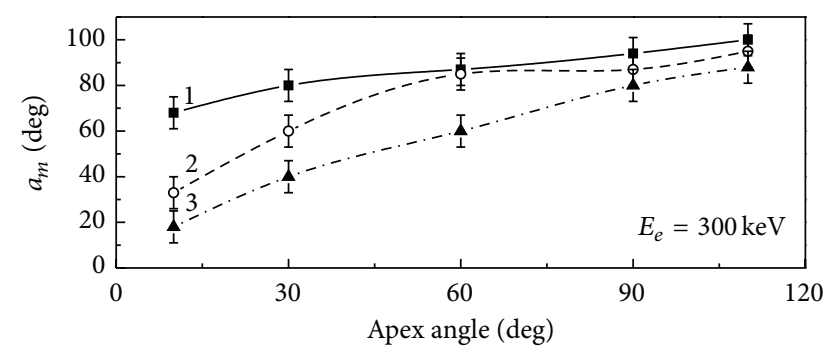

(a)

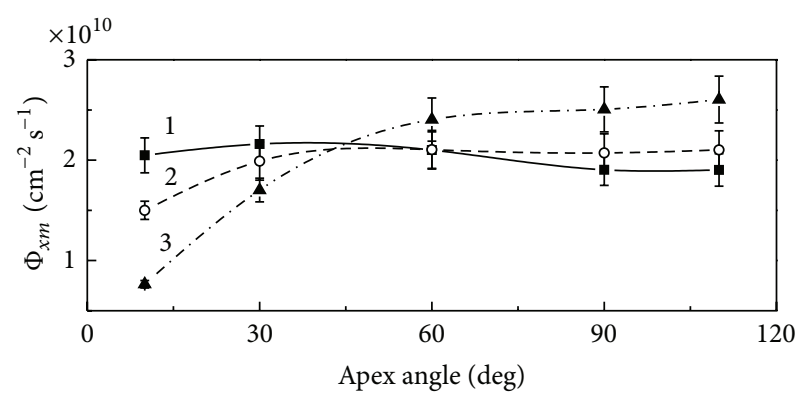

(b)

FIGURE 7: The dependences of the inclination angle, associated with the maximum value of the flux of generated X-rays (a), and the maximum value of the flux of generated X-rays (b) on the value of the apex angle of the conical anode at different electron beam radii: $0.5 \mathrm{~mm}(1), 2.0 \mathrm{~mm}(2)$, and $5.0 \mathrm{~mm}$ (3). The electron energy is $300 \mathrm{keV}$.

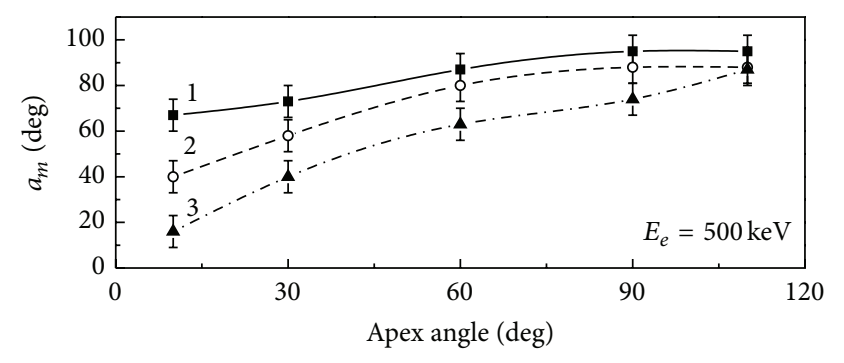

(a)

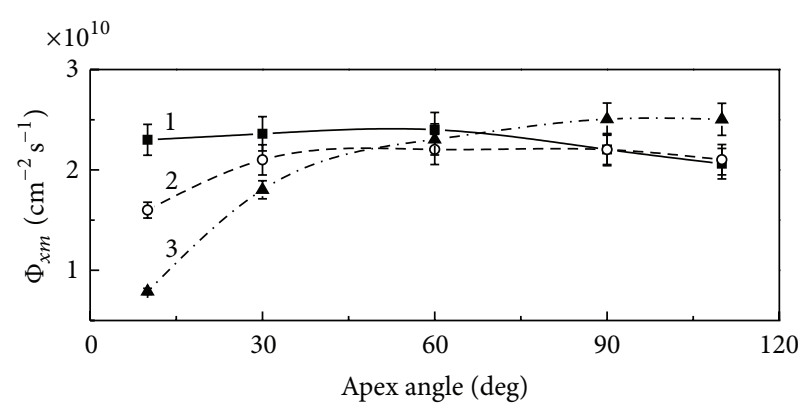

(b)

Figure 8: The dependences of the inclination angle, associated with the maximum value of the flux of generated X-rays (upper plot), and the maximum value of the flux of generated X-rays (lower plot) on the value of the apex angle of the conical anode at different electron beam radii: $0.5 \mathrm{~mm}(1), 2.0 \mathrm{~mm}(2)$, and $5.0 \mathrm{~mm}$ (3). The electron energy is $500 \mathrm{keV}$.

it is necessary to estimate the geometrical position of the cylindrical hole inside the collimator. Only photons whose coordinates lie on the hole on the first collimator surface and on the hole on the second collimator surface will be able to pass through the collimator hole. To minimise the statistical deviations in the calculated number of collimated photons, it is possible to simulate the circular slit instead of the hole. This estimate is acceptable because a cylindrical geometry is used, which is symmetrical about the $z$-axis in any direction. The number of photons will be greater with a circular slit and depend on the radius of the collimator hole $\left(r_{c}\right)$ and on the external radius of the collimator:

$$
\frac{\Delta N_{\text {slit }}}{\Delta N_{\text {hole }}} \approx \frac{2 \pi\left(R_{c}+\Delta R\right) 2 r_{c}}{\pi r_{c}^{2}}=\frac{4\left(R_{c}+\Delta R\right)}{r_{c}} .
$$

A simplified schematic of the measurement of the number of photons that can pass through the collimator slit (or hole) in the model is shown in Figure 9.

The translation of the photon coordinates on the second cylindrical surface was performed by using formulae (2), (3), and (4). As shown in Figure 9, the output X-ray beam (in direct projection through the collimator slit on the conical anode) was offset by a distance $2 r_{c} / \sin (\alpha)$ relative to the position of the top of the cone. The following logical conditions were used to select the photons with a momentum vector directed through the collimator slit. These logical conditions can be used only for $Z$-coordinates because for each photon located on the cylindrical surface with radius $R_{c}$, 


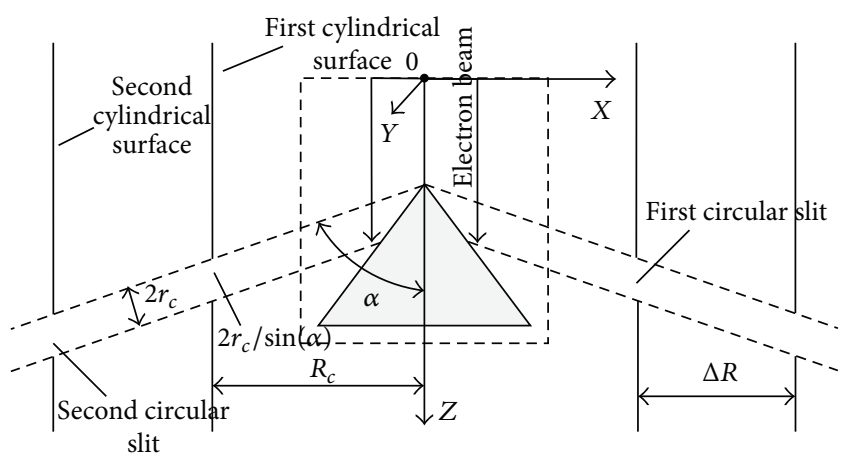

FIGURE 9: A schematic (side view) of the measurement of the number of photons (generated by MC) that can pass through the collimator slit.

the following relation is satisfied: $x_{j}^{2}+y_{j}^{2}=R_{c}^{2}$. The condition for the first cylindrical surface is as follows:

$$
Z_{c}+\frac{R_{c}}{\operatorname{tg}(\alpha)}<Z_{2 i}<Z_{c}+\frac{R_{c}}{\operatorname{tg}(\alpha)}+\frac{2 r_{c}}{\sin (\alpha)},
$$

where $Z_{c}$ is the coordinate of the top of the cone and $Z_{2 i}$ is the coordinate of the photon with index $i$ after the first coordinate translation onto the first cylindrical surface (from the initial square surface). The condition for the second cylindrical surface is similar:

$$
Z_{c}+\frac{R_{c}+\Delta R}{\operatorname{tg}(\alpha)}<Z_{3 i}<Z_{c}+\frac{R_{c}+\Delta R}{\operatorname{tg}(\alpha)}+\frac{2 r_{c}}{\sin (\alpha)}
$$

where $Z_{3 i}$ is the $Z$-coordinate of the photon with index $i$ after the second translation onto the second cylindrical surface with radius $R_{c}+\Delta R$. The following geometrical parameters of the external collimator were used for all simulations: $R_{c}=$ $10 \mathrm{~mm}, \Delta R=3.5 \mathrm{~mm}$, and $r_{c}=0.45 \mathrm{~mm}$. Typical results for the simulations of the beam intensity after collimation when the radius of the electron beam is $2.0 \mathrm{~mm}$ and the electron energy is $250 \mathrm{keV}$ are shown in Figure 10, with the electrical power of the electron beam normalised to 1.0 Watts.

It was observed that the shape of the function of the angular distribution of the photon intensity after collimation is different for different electron energies, radii of the electron beam, and shapes of the conical anode. The maximum value of the photon intensity in the collimated X-ray beam (at an inclination angle $\alpha_{j m}$ ) is one of the important parameters in this case. The relationships between these parameters are shown in Figures 11, 12, and 13 for different electron energies and geometries. A spline interpolation within the obtained statistical errors was used to connect the points on the presented figures. The errors in the inclination angles $\alpha_{j m}$ were estimated to be $2 \Delta \alpha=6^{\circ}$, and a step $\Delta \alpha$ was used to calculate the number of X-ray photons in the output beam after the collimation (using formulas (10) and (11)). The errors in the maximum values of the photon intensity $\left(I_{x m}\right.$, at an inclination angle $\alpha_{j m}$ ) were calculated as probable statistical deviations of the number of photons $\left(\Delta N_{j}\right)$ that were passed through the collimator using formula (5).

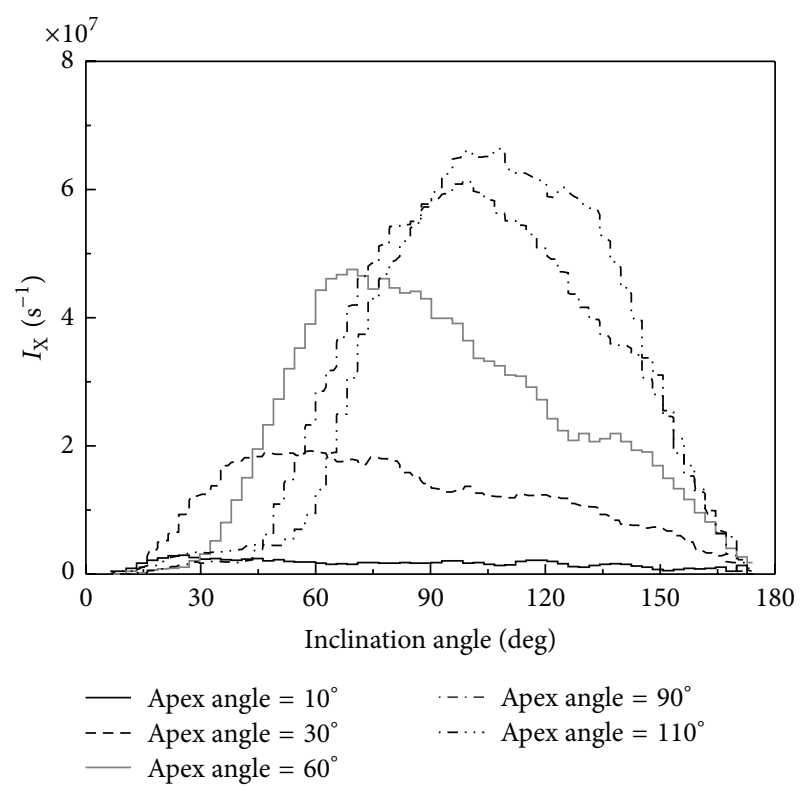

FIgURE 10: The angular distributions of the intensity of collimated Xray photons (through a collimator hole with radius $r_{c}$ ) in cylindrical geometry at different values of the apex angle of the anode when the electron energy is equal to $250 \mathrm{keV}$ and the electron beam radius is equal to $2.0 \mathrm{~mm}$.

The results, presented in Figures 11-13, allow for the selection of the optimal value of the anode apex angle or the inclination angle that maximises the intensity of the output X-ray beam if one of these parameters is known. The results (except for the precise value of the intensity after the collimation) hold for any collimator with the same ratios between geometrical parameters such as distance from the anode, the collimator thickness, and the diameter of the collimator hole. An accelerating potential in the range of 250 $500 \mathrm{kV}$ does not significantly affect the maximum flux of the generated X-rays at the same electrical power and at the same radial distance from the symmetry axis of the conical anode. The difference in the generated flux of X-rays is about $8 \%$ that can be explained by the additional attenuation of generated $\mathrm{X}$-rays in target media at electron energy of $500 \mathrm{keV}$.

3.4. The Efficiency of X-Ray Generation. High-energy electrons have different stopping probabilities for different thicknesses of the tungsten target and therefore have different probabilities of the generation of bremsstrahlung radiation. To determine the efficiency of generated and emitted X-ray photons, several simulations were carried out for electron energies equal to $250 \mathrm{keV}, 300 \mathrm{keV}$, and $500 \mathrm{keV}$. The $\mathrm{X}$-ray spectra for the accelerating potentials equal to $250 \mathrm{kV}$ and $500 \mathrm{kV}$ are shown in Figures 1 and 2. The efficiency coefficient for the energy transfer from an electron beam to the flux of generated and emitted X-rays for all directions over a solid angle $4 \pi$ was calculated using the following relation:

$$
\eta_{X} \approx \frac{\sum_{j}\left(\partial N_{X j} / \partial E \cdot \delta E \cdot E_{j}\right)}{N_{e 0} \cdot E_{e 0}},
$$




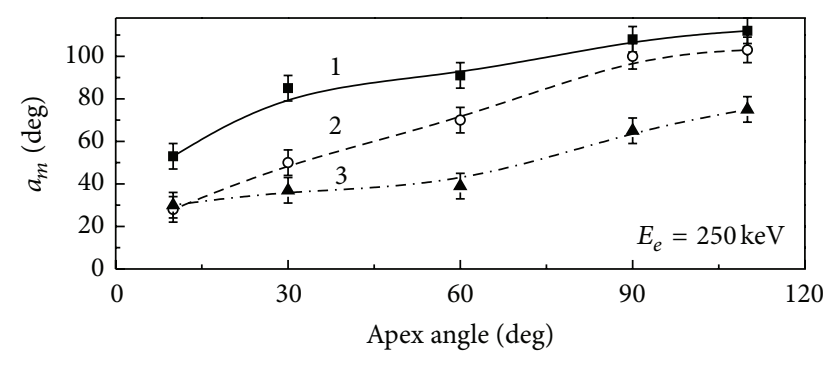

(a)

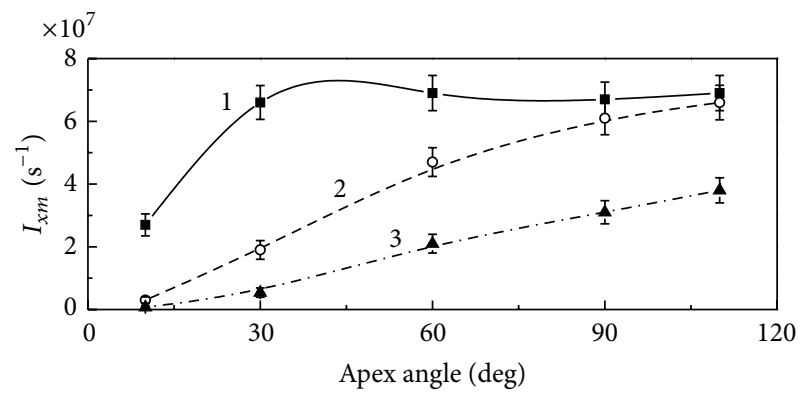

(b)

FIGURE 11: The dependences of the inclination angle, associated with the maximum value of the intensity of collimated X-rays (a), and of the maximum value of the intensity of collimated X-rays (b) on the value of the apex angle of the conical anode at an electron energy equal to $250 \mathrm{keV}$ and electron beam radii equal to $0.5 \mathrm{~mm}$ (1), $2.0 \mathrm{~mm}(2)$, and $5.0 \mathrm{~mm}(3)$.

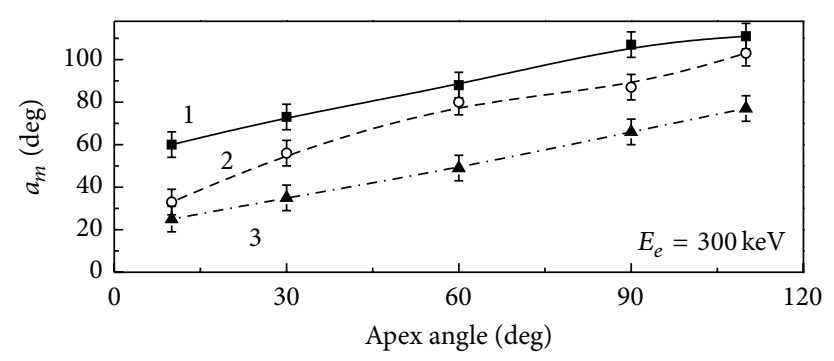

(a)

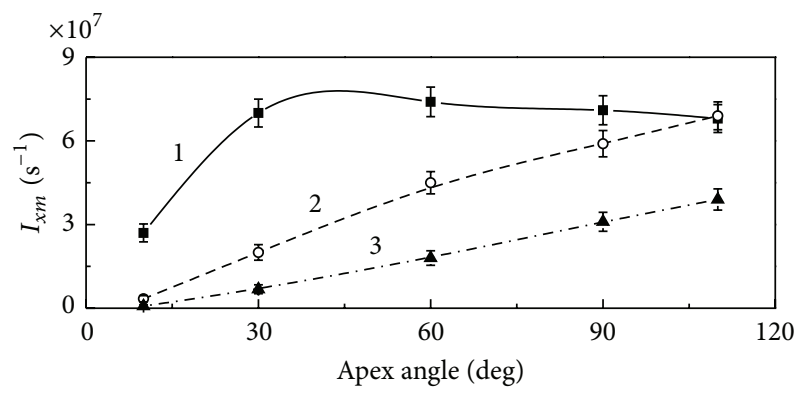

(b)

FIGURE 12: The dependences of the inclination angle, associated with the maximum value of the intensity of collimated X-rays (upper plot), and of the maximum value of the intensity of collimated $\mathrm{X}$ rays (lower plot) on the value of the apex angle of the conical anode at an electron energy equal to $300 \mathrm{keV}$ and electron beam radii equal to $0.5 \mathrm{~mm}(1), 2.0 \mathrm{~mm} \mathrm{(2)}$, and $5.0 \mathrm{~mm} \mathrm{(3).}$

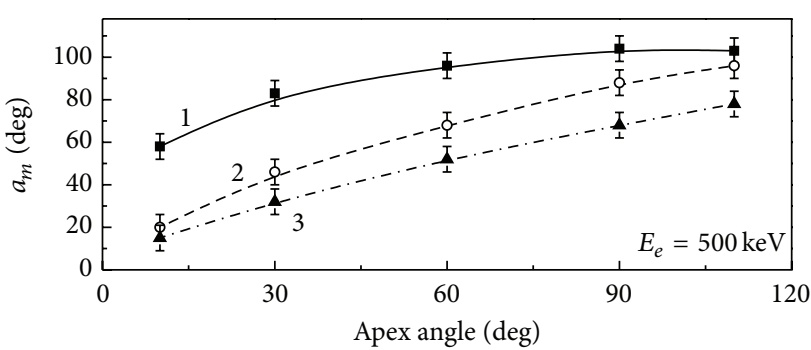

(a)

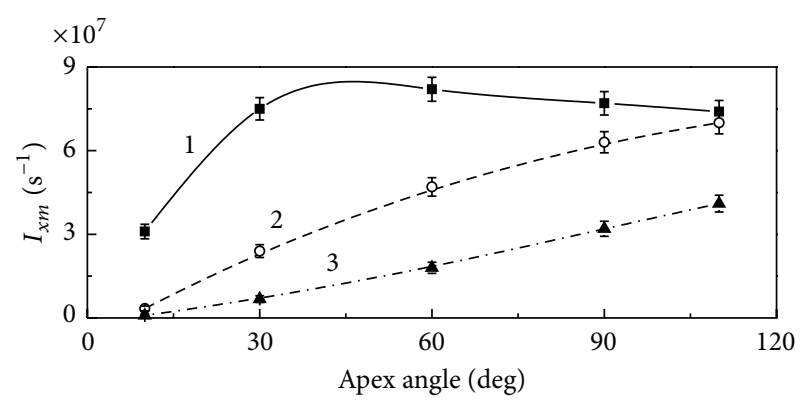

(b)

FIGURE 13: The dependences of the inclination angle, associated with the maximal value of the intensity of collimated X-rays (upper plot), and of the maximal value of the intensity of collimated X-rays (lower plot) on the value of the apex angle of the conical anode at an electron energy equal to $500 \mathrm{keV}$ and electron beam radii equal to $0.5 \mathrm{~mm}(1), 2.0 \mathrm{~mm}(2)$, and $5.0 \mathrm{~mm}$ (3).

where $\delta E=2 \mathrm{keV}$ is the energy step of the simulated X-ray spectrum, which has energy distribution $\partial N_{X j} / \partial E$, and $N_{e 0}$ is the initial number of high-energy electrons with energy $E_{e 0}$. Formula (12) takes into account only photons unabsorbed in the target. The error in the efficiency coefficient was calculated using the following relation [12]:

$$
\delta \eta_{X}=\frac{1}{N_{e 0} E_{e 0}} \sqrt{\sum_{j}\left(\sqrt{\frac{\partial N_{X j}}{\partial E} \delta E} \cdot E_{j}\right)^{2}} .
$$

The calculated efficiency coefficients of the energy transfer from the electron beam to the flux of generated and emitted X-rays are shown in Figures 14 and 15 for electron energies equal to $250 \mathrm{keV}$ and $500 \mathrm{keV}$ and for different electron beam radii and apex angles of the conical anode. The theoretical coefficient of total efficiency of X-ray generation was calculated and plotted as well to compare with the efficiency of the output flux of emitted photons. This comparison can help to estimate how the different shapes of the conical anode influence the additional attenuation of the generated photons.

As shown in Figures 14 and 15, an X-ray tube with a conical target shows relatively low X-ray generation efficiency compared to the total efficiency of the generation of bremsstrahlung radiation $[13,15]$. The results obtained for different electron energies demonstrate that the additional attenuation inside the target is the main reason for this effect, 


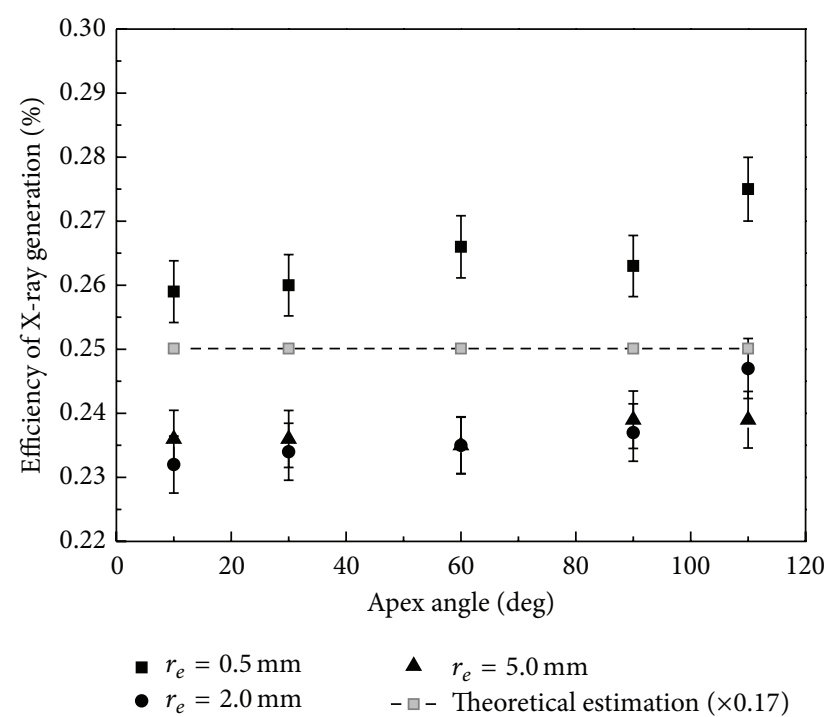

FIGURE 14: The dependences of the efficiency coefficients of the energy transfer from the electron beam to the flux of generated and emitted X-ray photons on the apex angle of the conical anode for different electron beam radii and at an electron energy equal to $250 \mathrm{keV}$.

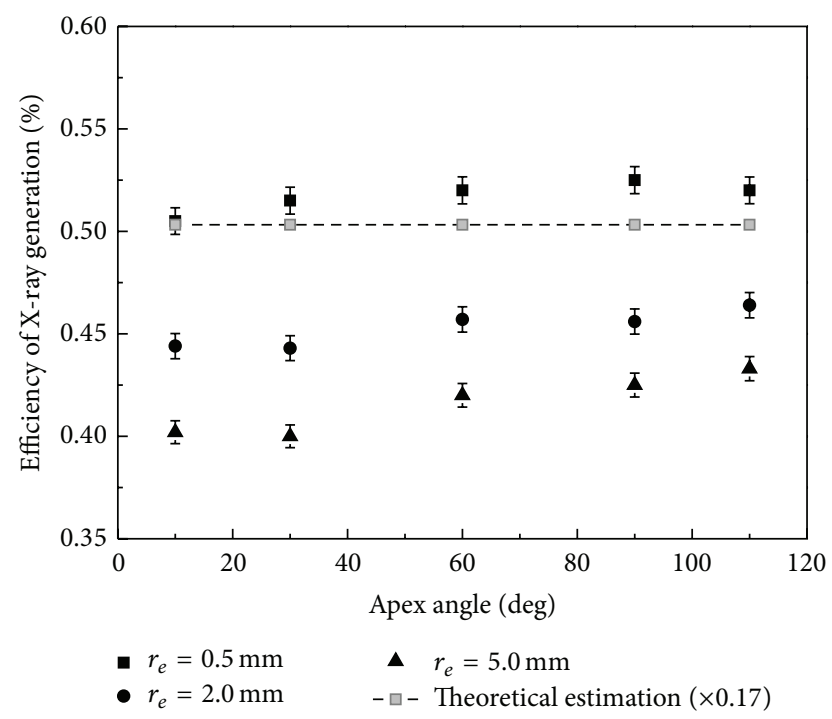

FIgURE 15: The dependences of the efficiency coefficients of the energy transfer from the electron beam to the flux of generated and emitted X-ray photons on the apex angle of the conical anode for different electron beam radii and at an electron energy equal to $500 \mathrm{keV}$.

which clearly depends on the range of electron energies in the target medium [14].

\section{Conclusions}

From Monte Carlo simulations of the X-ray generation process in an X-ray tube with a conical target, several important parameters and dependences were determined: the X-ray intensity of generated photons in the energy range of 250 $500 \mathrm{keV}$, the optimal target shape, the angular distribution of the intensity of generated X-rays as well as photons collimated through the cylindrical hole of an external collimator, and the efficiency coefficient for energy transfer from an electron beam to a generated X-ray flux. It was demonstrated that the angular distribution of the flux of generated X-rays and the angular distribution of the intensity in a collimated Xray beam depend on the radius of the electron beam and on the shape of the conical target and that these dependences are different at different accelerating potentials of the X-ray tube. The optimal values of the geometrical parameters of a scanning system based on an X-ray tube integrated with an external collimator were observed to maximise the output $\mathrm{X}$-ray intensity in the beam. These results can be used in practical applications to design scanning systems based on the pencil-beam technique and X-ray tubes with massive conical targets.

\section{Conflict of Interests}

The authors declare that there is no conflict of interests regarding the publication of this paper.

\section{Acknowledgment}

This work was partially funded by the Research Council of Norway under Contract 200888.

\section{References}

[1] G. Harding and J. Kosanetzky, "Scattered X-ray beam nondestructive testing," Nuclear Instruments and Methods in Physics Research Section A: Accelerators, Spectrometers, Detectors and Associated Equipment, vol. 280, pp. 517-528, 1989.

[2] D. N. Zeiger, J. Sun, G. E. Schumacher, and S. Lin-Gibson, "Evaluation of dental composite shrinkage and leakage in extracted teeth using X-ray microcomputed tomography," Dental Materials, vol. 25, no. 10, pp. 1213-1220, 2009.

[3] L. Goldstein, S. O. Prasher, and S. Ghoshal, “Three-dimensional visualization and quantification of non-aqueous phase liquid volumes in natural porous media using a medical X-ray Computed Tomography scanner," Journal of Contaminant Hydrology, vol. 93, no. 1-4, pp. 96-110, 2007.

[4] M. Lindner, L. Blanquart, P. Fischer, H. Krüger, and N. Wermes, "Medical X-ray imaging with energy windowing," Nuclear Instruments and Methods in Physics Research A: Accelerators, Spectrometers, Detectors and Associated Equipment, vol. 465, no. 1, pp. 229-234, 2001.

[5] L. Auditore, R. C. Barna, U. Emanuele, D. Loria, A. Trifiro, and M. Trimarchi, "X-ray tomography system for industrial applications," Nuclear Instruments and Methods in Physics Research $B$, vol. 266, no. 10, pp. 2138-2141, 2008.

[6] R. D. Luggar, E. J. Morton, P. M. Jenneson, and M. J. Key, "X-ray tomographic imaging in industrial process control," Radiation Physics and Chemistry, vol. 61, no. 3-6, pp. 785-787, 2001.

[7] A. Ihsan, S. H. Heo, H. J. Kim, C. M. Kang, and S. O. Cho, "An optimal design of X-ray target for uniform X-ray emission from an electronic brachytherapy system," Nuclear Instruments and 
Methods in Physics Research B, vol. 269, no. 10, pp. 1053-1057, 2011.

[8] G. Harding, B. Jordan, and J. Kosanetzky, "A new fluorescent $\mathrm{X}$-ray source for photon scattering investigations," Physics in Medicine and Biology, vol. 36, no. 12, article 3, pp. 1573-1583, 1991.

[9] S. Henzell, S. S. Dhaliwal, R. I. Price et al., "Comparison of pencil-beam and fan-beam DXA systems," Journal of Clinical Densitometry, vol. 6, no. 3, pp. 205-210, 2003.

[10] S. Humphries, "Computational techniques in xenosintegrated 3D software suite for electron and X-ray physics," in Proceedings of the IEEE 34th International Conference on Plasma Science (ICOPS '07), p. 729, Albuquerque, NM, USA, June 2007.

[11] F. Salvat, J. M. Fernández-Varea, and J. Sempau, PENELOPE2011: A Code System for Monte Carlo Simulation of Electron and Photon Transport, OECD Nuclear Energy Agency, 2011.

[12] J. R. Taylor, An Introduction to Error Analysis, University Science Books, 1996.

[13] S. A. Ivanov and G. A. Shchukin, Rentgenovskie Trubki Tekhnicheskogo Naznacheniya (X-ray Tubes for Technical Purposes), Energoatomizdat, Leningrad, Russia, 1989, (Russian).

[14] K. N. Mukhin, Nuclear Physics, Macdonald \& Co., 1970.

[15] H. W. Koch and J. W. Motz, "Bremsstrahlung cross-section formulas and related data," Reviews of Modern Physics, vol. 31, no. 4, pp. 920-955, 1959. 

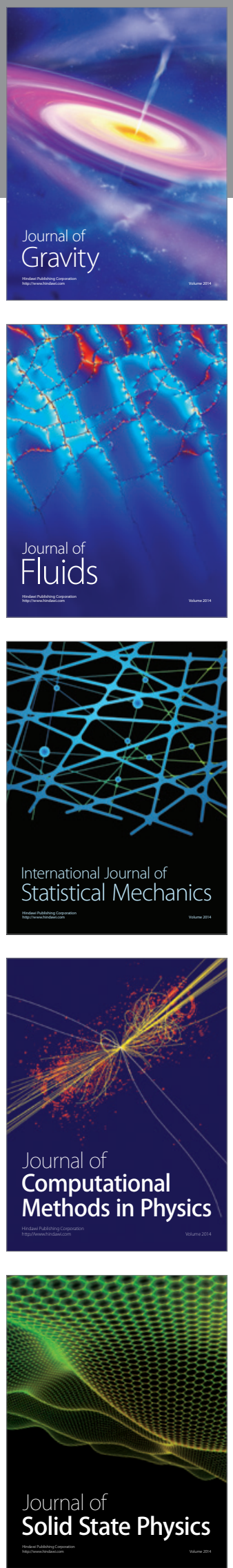

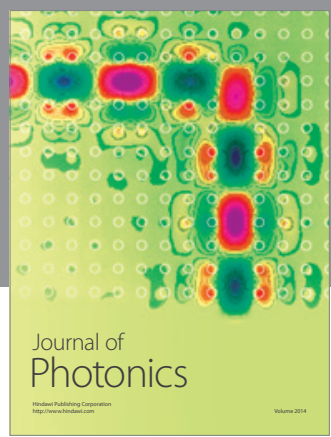

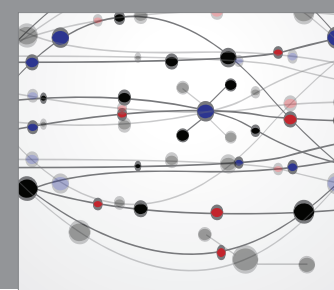

The Scientific World Journal

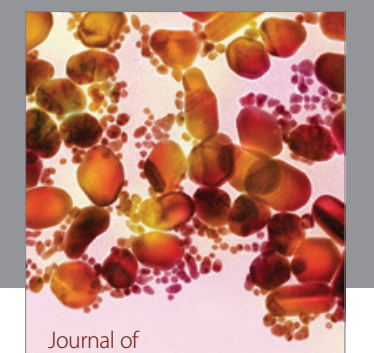

Soft Matter
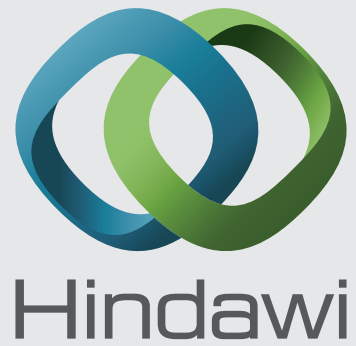

Submit your manuscripts at

http://www.hindawi.com
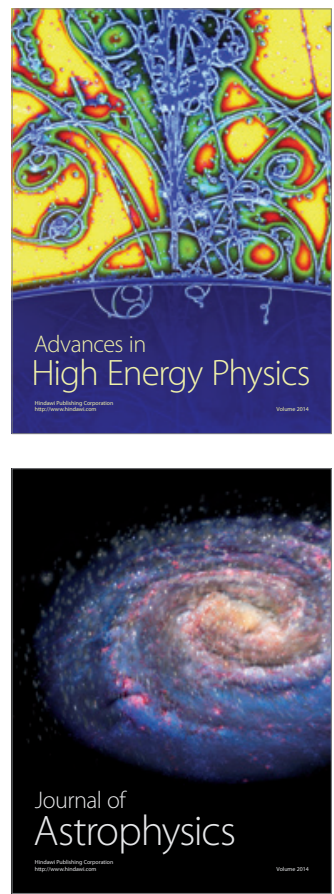
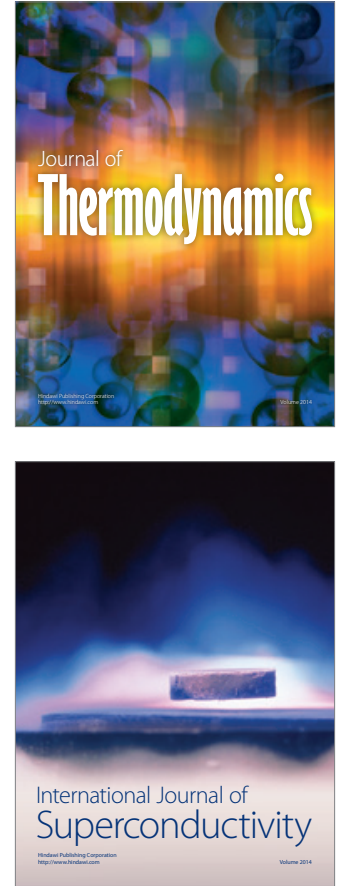
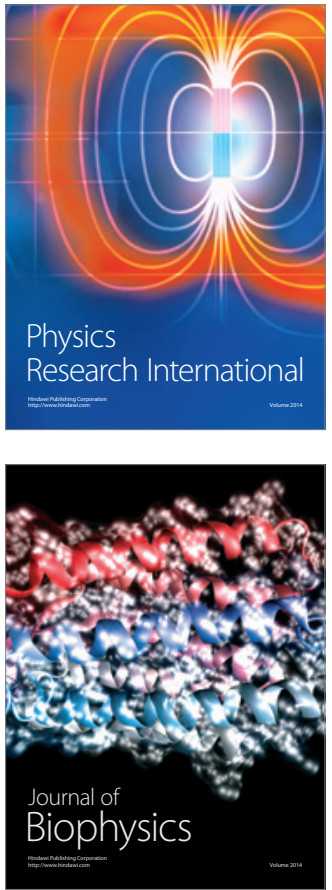
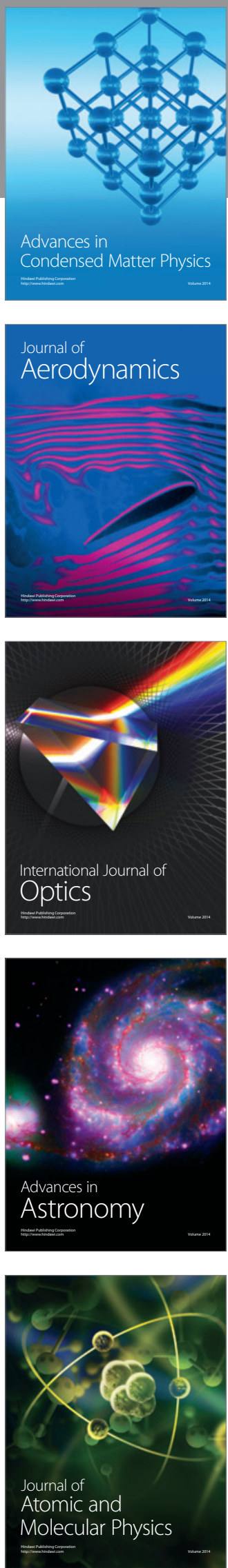